\title{
Interacting Bosons at Finite Angular Momentum Via Complex Langevin
}

\author{
Casey E. Berger* \\ Department of Physics and Astronomy, University of North Carolina, Chapel Hill, NC, 27599, \\ USA \\ E-mail: cberger3elive.unc.edu

\section{Joaquín E. Drut} \\ Department of Physics and Astronomy, University of North Carolina, Chapel Hill, NC, 27599, \\ USA \\ E-mail: druteemail.unc.edu
}

\begin{abstract}
Quantum field theories with a complex action suffer from a sign problem in stochastic nonperturbative treatments, making many systems of great interest - such as polarized or mass-imbalanced fermions and QCD at finite baryon density - extremely challenging to treat numerically. Another such system is that of bosons at finite angular momentum; experimentalists have successfully achieved vortex formation in ultracold bosonic atoms, and have measured quantities of interest such as density profiles and the moment of inertia. However, the treatment of superfluids requires the use of complex bosons, making the usual numerical methods unusable. In this work, we apply complex Langevin, a method that has gained much attention in lattice $\mathrm{QCD}$, to the calculation of basic properties of interacting bosons at finite angular momentum. We show preliminary results for the angular momentum and moment of inertia and benchmark calculations in the noninteracting limit.
\end{abstract}

The 36th Annual International Symposium on Lattice Field Theory - LATTICE2018 22-28 July, 2018

Michigan State University, East Lansing, Michigan, USA.

\footnotetext{
*Speaker.
} 


\section{Introduction}

Nonrelativistic bosons at finite angular momentum have been studied experimentally using ultracold bosonic atoms. These systems - rotating superfluids and Bose-Einstein condensates can provide insights across disciplines of physics, including into rotating nuclei, pulsars, and the behavior of superconductors in a magnetic field. In 1949, Lars Onsager predicted that vortices would form in rotating superfluids [1]. Another 30 years after these predictions, the first direct observation of quantum vortices was made in rotating superfluid ${ }^{4} \mathrm{He}$ [2].

Since then, work on ultracold rotating bosonic atoms has achieved single vortex and multivortex lattice formation and has studied the properties of these systems across temperature regimes and at the superfluid transition [3]. In 2000, vortex formation was observed in stirred, magneticallytrapped ${ }^{87} \mathrm{Rb}$ atoms [4]. The next year, the Ketterle Group at MIT observed triangular vortex lattices of up to 130 vortices in rotating ultracold sodium atoms [5]. Ultracold atoms provide a highly controlled, tuneable setting for studying vortex formation and other properties of rotating superfluids.

Theoretically, treatment of these systems has stalled due to the presence of the sign problem. Contrary to what is common knowledge for relativistic systems, a finite chemical potential does not itself induce a sign problem in nonrelativistic bosons. Indeed, it is the asymmetry in the temporal derivative that makes the action complex in the case of complex bosons, as we show below; real bosons do not have this problem. Complex bosons are required, in fact, to describe the behavior of superfluids, as the superflow velocity is the gradient of the phase of the complex field. In other words: superfluidity (as superconductivity) is a fundamentally complex phenomenon. On the other hand, it is worth noting that nonrelativistic fermions at finite chemical potential do not feature a sign problem for attractive interactions and vanishing polarization; finite angular momentum, however, does induce a sign problem by virtue of the breaking of time-reversal invariance. Thus a sign problem (specifically a complex phase problem) must be overcome when studying the formation of vortices in superfluid systems, regardless of whether they are bosonic or fermionic.

Rotating superfluids exist in important physical systems that cut across vastly different scales in physics. They are of interest in condensed matter in order to understand the effect of a magnetic field on superconductors; in nuclear physics for describing the behavior of rotating nuclei; and in astrophysics for illuminating the dynamics of neutron stars and pulsars. To continue to progress in these areas of physics, a method to work around the sign problem must be used. This work takes a step in that direction by applying one such method: the complex Langevin (CL) algorithm.

\section{Methods}

\subsection{Complex Langevin Formalism}

Complex Langevin is a method put forward independently by Parisi [6] and Klauder [7], which extends the idea of stochastic quantization (SQ) to complex variables. The SQ approach to quantum field theory for a real field $\phi(x)$ (where $x$ contains both space and time) governed by a real action $S[\phi]$ proceeds via a stochastic differential equation. This equation evolves the system in a 'fictitious' time $t$ such that if equilibrium is reached as $t \rightarrow \infty$, the noise-averaged correlation functions (at equal fictitious time) approach the corresponding correlation functions of the target 
quantum field theory. This is known as the Langevin process, and the equation that governs it is the Langevin equation,

$$
\delta \phi(x ; t)=K_{x} \delta t+\eta_{x}(t),
$$

where $K_{x}=-\frac{\delta S}{\delta \phi(x)}$ is the drift, and $\eta_{x}$ is a Gaussian white noise variable with $\langle\eta\rangle=0$ and $\left\langle\eta^{2}\right\rangle=$ $2 \delta t$, where the angle brackets denote average over $\eta$.

The Langevin method mentioned above is a well-established method for real-valued variables, $\phi$ on a real manifold $\mathscr{M}$. The solutions $\phi(t)$ of this equation will vary depending on the white noise introduced, but they will be distributed according to a probability distribution, $P(\phi ; t)$, which we can use to average an observable of interest, $\mathscr{O}[\phi]$, in the usual way:

$$
\langle\mathscr{O}\rangle=\int \mathscr{D} \phi P(\phi ; t) \mathscr{O}[\phi] .
$$

In practice, the above expectation value is estimated as in other stochastic methods by averaging $\mathscr{O}[\phi]$ over realizations of the field $\phi$ obtained throughout the Langevin evolution.

The idea behind CL is that at least for certain classes of theories and correlation functions (i.e. observables), it is possible to perform calculations by extending the real stochastic process of Eq. (2.1) into a complex one. Thus, for a theory governed by a complex action $S[\phi]$ (where a step in the Langevin process would result in an imaginary component for $\phi$ ), CL extends the target manifold of $\phi(x)$ to the complex plane by setting $\phi(x) \rightarrow \phi_{R}(x)+i \phi_{I}(x)$, and analytically extending the domain of the action functional, i.e. $S[\phi] \rightarrow S\left[\phi_{R}(x)+i \phi_{I}(x)\right]$. For this extension to be valid, $S\left[\phi_{R}(x)+i \phi_{I}(x)\right]$ must be a holomorphic function. With such extensions, the CL method proceeds very much in the same way as SQ, but now with a double system of stochastic differential equations, which we will write explicitly below for the problem at hand.

This method has enjoyed a recent renaissance in Lattice QCD, after it was shown to be effective in models of finite density QCD that exhibit a sign problem [8]. It has been shown to overcome the Silver Blaze problem in the relativistic Bose gas with finite chemical potential [9, 10]. Even more recently, the method has been shown to be effective in $1+1$ dimensional QCD in the thermodynamic limit [11]. When applied to chiral random matrix theory at nonzero chemical potential, it produces results which agree with analytical results at large quark mass - although not for small quark mass [12]. Similarly, it has been able to compute the QCD phase diagram in the heavy quark limit on the entire temperature-chemical potential plane [13].

Outside of relativistic physics, CL is just emerging as a useful method. A 2015 paper discusses the results of applying CL to bosonic quantum field theory in a rotating frame to circumvent the sign problem [14]. More recently, work on fermionic systems in one spatial dimension calculated the density and pressure equations of state, comparing CL results with third-order lattice perturbation theory [15]. That work was recently extended to polarized systems [16]. A parallel line of research pursued the calculation of high-order virial coefficients using the particle projection method via CL [17]. The same methods were applied to the polarized unitary Fermi gas at finite temperature [18]. The ground state energy of mass-imbalanced fermions was computed via CL and showed agreement with other methods [19]. Furthermore, the results obtained by CL in that work demonstrated better-controlled statistical uncertainties and results were obtained beyond the regimes accessible by other methods. 


\subsection{Complex Langevin for NonRelativistic Bosons with Finite Angular Momentum}

The action for a non-relativistic system in $2+1$ Euclidean dimensions follows the general form

$$
S=\int d^{2} x d \tau\left[\phi^{*}\left(\mathscr{H}-\mu-\omega_{z} L_{z}\right) \phi+\lambda\left(\phi^{*} \phi\right)^{2}\right],
$$

where $\mathscr{H}=\partial_{\tau}-\frac{\nabla^{2}}{2 m}, L_{z}=i\left(x \partial_{y}-y \partial_{x}\right)$, and $\lambda$ represents a contact interaction coupling.

Using a backward-difference derivative, we discretize this action to a $2+1$ dimensional lattice with spatial lattice spacing $a=1$ and temporal lattice spacing $d \tau=0.2$ (in units set by $a$ ). We impose hard-wall boundary conditions in the spatial directions and periodic boundary conditions in imaginary time.

In order to treat the complex fields within the CL method, we first write the field as the complex sum of two real fields: $\phi=\frac{1}{\sqrt{2}}\left(\phi_{1}+i \phi_{2}\right)$, and then complexify those real fields, $\phi_{a}=\phi_{a}^{R}+i \phi_{a}^{I}$. Once the fields are complexified, the drift function can be derived for the four components of the fields. The fields evolve according to the CL equation, with a drift function derived from the the action and a Gaussian-distributed real noise:

$$
\begin{aligned}
& \phi_{a, r}^{R}(n+1)=\phi_{a, r}^{R}(n)+\varepsilon K_{a, r}^{R}(n)+\sqrt{\varepsilon} \eta_{a, r}(n), \\
& \phi_{a, r}^{I}(n+1)=\phi_{a, r}^{I}(n)+\varepsilon K_{a, r}^{I}(n)
\end{aligned}
$$

where $a=1,2$. The drift functions, $K$, are derived from the action:

$$
\begin{aligned}
& K_{a, r}^{R}=-\operatorname{Re}\left[\left.\frac{\delta S}{\delta \phi_{a, r}}\right|_{\phi_{a} \rightarrow \phi_{a}^{R}+i \phi_{a}^{I}}\right], \\
& K_{a, r}^{I}=-\operatorname{Im}\left[\left.\frac{\delta S}{\delta \phi_{a, r}}\right|_{\phi_{a} \rightarrow \phi_{a}^{R}+i \phi_{a}^{I}}\right],
\end{aligned}
$$

and are defined at each site on the spacetime lattice. The drift functions evolve the fields at each step in Langevin time.

\section{Results}

The formulation of this problem using complex fields results in a sign problem. Most previous work on CL in bosonic systems has been for relativistic actions (see e.g. [9, 10, 20]). Such a relativistic Bose gas has spatial and temporal derivatives which both result in symmetric matrices when evaluated on the lattice (at vanishing chemical potential). That symmetry ensures that the original action $S[\phi]$ can be written in terms of the real and imaginary parts of the field $\left(\phi_{1}, \phi_{2}\right)$, with corresponding actions that are explicitly real and completely decoupled. In the nonrelativistic case, on the other hand, the time derivative component is not symmetric, such that the action is explicitly complex when written in terms of $\phi_{1}$ and $\phi_{2}$. The resulting discretized form of the action therefore suffers from a sign problem, even before the introduction of any additional elements which usually complicate the problem (such as chemical potential or angular momentum).

Naturally, the free Bose gas is an analytically solvable system (even on the lattice and with hard-wall boundary conditions) against which we can check our methods. Since a sign problem has already arisen due to the formulation of the action using complex fields, this is a non-trivial 
check (in contrast with its fermionic analogue) which we can use to test our CL implementation. To that end, we computed the density for the free Bose gas in $2+1$ dimensions for lattices of length $N_{x}=10,20$ in each spatial direction and $N_{\tau}=6,10$ in the imaginary time direction, and the $\mathrm{CL}$ results showed good agreement with the analytical solution, as seen in Fig. 1. We note that small lattice sizes affect the behavior with the CL step size $d t_{C L}$ : the large-volume data uniformly approaches the exact solution; the small-volume data displays the opposite behavior.
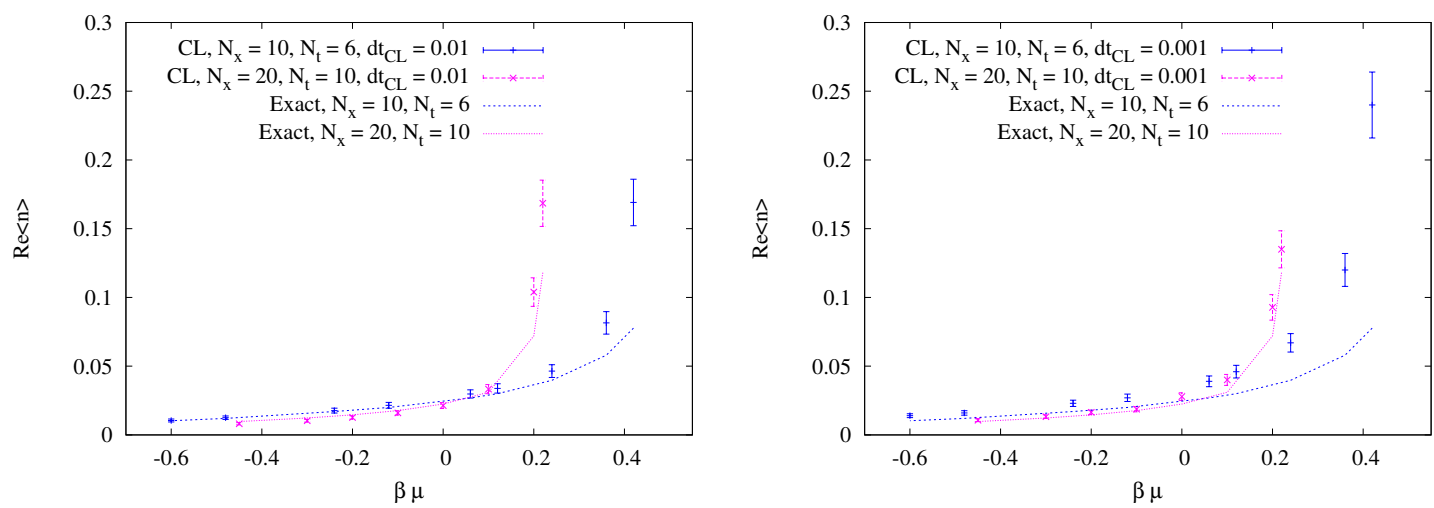

Figure 1: Density equation of state of the free Bose gas in $2+1$ dimensions, as a function of $\beta \mu$, obtained via CL. The left panel shows the real part of the CL result along with the analytic answer for a CL time step $d t_{C L}=0.01$; the right panel shows the same quantities for $d t_{C L}=0.001$.

Having shown the viability of the method and tested the implementation, we continue to compute quantities of interest for the rotating, interacting system. In the left panel Fig. 2, we show preliminary results for the real part of the angular momentum of a rotating, interacting system at nonzero chemical potential. We see the expected linear dependence of the angular momentum on the rotation frequency.The preliminary results for the imaginary part of $\left\langle L_{z}\right\rangle$ are shown in the lower panel of Fig. 2; these results are consistent with zero, as expected.
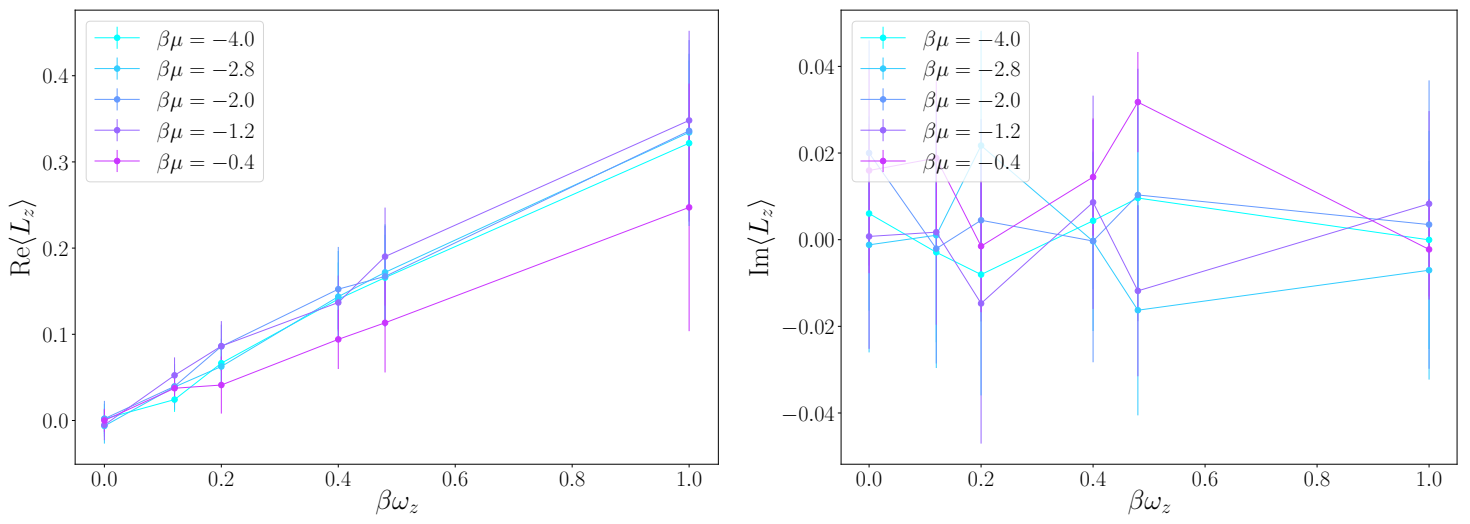

Figure 2: Expectation value of $L_{z}$ as a function of the (dimensionless) angular velocity $\beta \omega_{z}$ for several values of the (dimensionless) chemical potential $\beta \mu$. Left panel shows the real part of the CL result; right panel shows the imaginary part (note the difference in scale in the vertical axis).

The moment of inertia was also computed for the same parameter values as those of Fig. 2, and the results are shown in Fig. 3. The left panel shows the real part of the moment of inertia, which 
appears to display the expected constant behavior. As the value of $\beta \omega$ increases, the moment of inertia remains approximately constant, in agreement with the expectation from Fig. 2. Finally, the
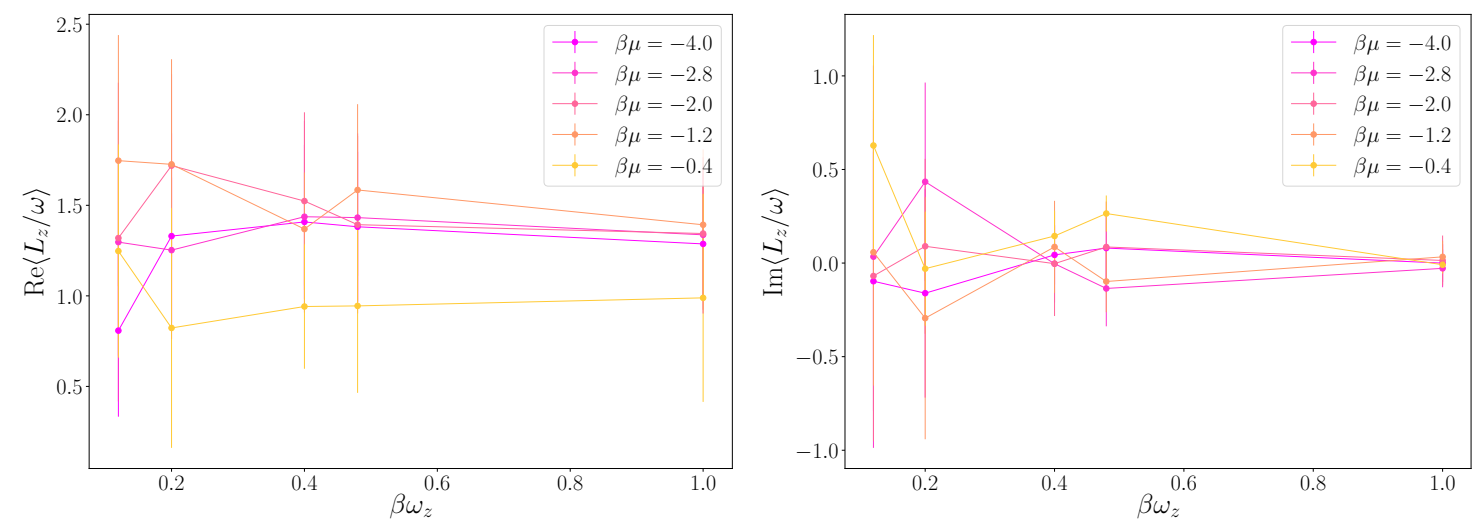

Figure 3: Moment of inertia $I_{z} \equiv L_{z} / \omega_{z}$ as a function of the (dimensionless) angular velocity $\beta \omega_{z}$ for several values of the (dimensionless) chemical potential $\beta \mu$. Left panel shows the real part of the CL result; right panel shows the imaginary part (note the difference in scale in the vertical axis).

imaginary part of the moment of inertia is shown in the right panel of Fig. 3, and also is statistically zero. The results shown here for $\left\langle L_{z}\right\rangle$ and $I_{z}$ show a substantial amount of statistical noise. Further investigation of these quantities for lower temperatures and with improved statistics is underway; we therefore regard the results shown here as preliminary at best.

\section{Summary and conclusion}

In this contribution we have shown our first implementation of the CL algorithm for nonrelativistic bosons at finite temperature, chemical potential, and angular momentum. We have explained how the bosonic case is very different from its fermionic counterpart in the sense that the sign (complex phase) problem appears already in the absence of interaction, chemical potential, or angular momentum. To test out CL implementation, we used the noninteracting result on the lattice with hard-wall boundary conditions. We then showed preliminary results for a system of interacting $2+1$ dimensional bosons at finite angular momentum, for which we extracted the expectation value of $L_{z}$ and the moment of inertia as a function of the angular velocity. It is worth noting that previous works have explored the behavior of bosons at finite angular momentum with $\mathrm{CL}$, as applied to the (3+1)-dimensional problem [14]. The results shown in the previous section are promising, and further study is underway. Current calculations are being performed for smaller values of $\beta \mu$ in order to ensure that the superfluid regime is achieved with the objective of studying the effects of superfluidity on the angular momentum, density, and moment of inertia. This regime will exhibit some of the unusual behavior that has already been shown through experiments, and which we expect to reproduce through our CL simulations.

\section{References}

[1] L. Onsager, Statistical hydrodynamics, Il Nuovo Cimento (1943-1954) 6 (1949) 279. 
[2] E. J. Yarmchuk, M. J. V. Gordon and R. E. Packard, Observation of stationary vortex arrays in rotating superfluid helium, Phys. Rev. Lett. 43 (1979) 214.

[3] A. L. Fetter, Rotating trapped bose-einstein condensates, Rev. Mod. Phys. 81 (2009) 647.

[4] K. W. Madison, F. Chevy, W. Wohlleben and J. Dalibard, Vortex formation in a stirred bose-einstein condensate, Phys. Rev. Lett. 84 (2000) 806.

[5] J. R. Abo-Shaeer, C. Raman, J. M. Vogels and W. Ketterle, Observation of vortex lattices in bose-einstein condensates, Science 292 (2001) 476 [http://science.sciencemag.org/content/292/5516/476.full.pdf].

[6] G. Parisi, On complex probabilities, Physics Letters B 131 (1983) 393 .

[7] J. R. Klauder, A Langevin approach to fermion and quantum spin correlation functions, Journal of Physics A Mathematical General 16 (1983) L317.

[8] G. Aarts and I.-O. Stamatescu, Stochastic quantization at finite chemical potential, JHEP 09 (2008) 018 [0807.1597].

[9] G. Aarts, Can Stochastic Quantization Evade the Sign Problem? The Relativistic Bose Gas at Finite Chemical Potential, Physical Review Letters 102 (2009) 131601 [0810 . 2089].

[10] G. Aarts, Complex langevin dynamics at finite chemical potential: mean field analysis in the relativistic bose gas, Journal of High Energy Physics 2009 (2009) 052.

[11] G. Aarts and K. Splittorff, Degenerate distributions in complex langevin dynamics: one-dimensional qcd at finite chemical potential, Journal of High Energy Physics 2010 (2010) 17.

[12] A. Mollgaard and K. Splittorff, Complex Langevin Dynamics for chiral Random Matrix Theory, Phys. Rev. D88 (2013) 116007 [1309.4335].

[13] G. Aarts, F. Attanasio, B. Jäger, E. Seiler, D. Sexty and I.-O. Stamatescu, Insights into the heavy dense QCD phase diagram using Complex Langevin simulations, PoS LATTICE2015 (2016) 155 [1510.09100].

[14] T. Hayata and A. Yamamoto, Complex langevin simulation of quantum vortices in a bose-einstein condensate, Phys. Rev. A 92 (2015) 043628.

[15] A. C. Loheac and J. E. Drut, Third-order perturbative lattice and complex langevin analyses of the finite-temperature equation of state of nonrelativistic fermions in one dimension, Phys. Rev. D 95 (2017) 094502.

[16] A. C. Loheac, J. E. Drut and J. Braun, Polarized fermions in one dimension: density and polarization from complex langevin calculations, perturbation theory, and the virial expansion., Phys. Rev. D 98 (2018) 054507.

[17] C. R. Shill and J. E. Drut, Virial coefficients of $1 d$ and $2 d$ fermi gases by stochastic methods and a semiclassical lattice approximation., Phys. Rev. A - in print (2018).

[18] L. Rammelmüller, A. Loheac, J. E. Drut and J. Braun, Finite-temperature equation of state of polarized fermions at unitarity, Phys. Rev. Lett. 121 (2018) 173001.

[19] L. Rammelmüller, W. J. Porter, J. E. Drut and J. Braun, Surmounting the sign problem in nonrelativistic calculations: A case study with mass-imbalanced fermions, Phys. Rev. D 96 (2017) 094506.

[20] C. Gattringer and K. Langfeld, Approaches to the sign problem in lattice field theory, International Journal of Modern Physics A 31 (2016) 1643007 [1603.09517]. 